\section{P-151 EMBEDDING DIGNITY INTO PRACTICE}

Emily Dobson. Saint Michael's Hospice, Harrogate, UK

\subsection{6/bmjspcare-2019-HUKNC.173}

Introduction The national commitments framework focussed on six key themes. Within these we decided to work on one 'maximising comfort and wellbeing' through the lens of dignity. We undertook a scoping exercise where we reviewed ten different patient notes to look at what we understand dignity to be and how this may be translated for patients, relatives, friends and carers. We identified that there were six tenets which relate to a person's feeling of dignity in the inpatient unit:

- Pain and symptom control;

- Loved ones cared for;

- Pragmatic concerns taken care of e.g. housing;

- Place of care established - often in line with preferred place of death;

- Control - being able to exercise choices made;

- Respect for choices made.

We identified that these six tenets working in tandem enabled a person to consider that they have dignity at the end of their life. Based on the identification of these six tenets we drew together a project.

Objectives

- value the uniqueness of every individual;

- uphold the responsibility to shape care around each individual;

- value communicating with individuals that are meaningful to them;

- recognise and respect how an individual's dignity may be affected when supported with their personal care;

- recognise that that an individual's surroundings and environment are important to their sense of dignity;

- value workplace cultures that actively promote the dignity of everybody;

- recognise the need to challenge care that may reduce the dignity of the individual.

Current workplans

- Adapting our care plans;

- To disseminate training on Transactional Analysis;

- To create a policy/procedure around challenging dignity which would allow care plans to be challenged where they do not feed into the established six tenets identified above.

\section{P-152 PERSON-CENTRED CARE: FEASIBILITY AND ACCEPTABILITY OF THE SUPPORT NEEDS APPROACH FOR PATIENTS (SNAP)}

${ }^{1}$ Carole Gardener, 'Gail Ewing, ${ }^{2}$ Morag Farquhar. 'University of Cambridge, Cambridge, UK; ${ }^{2}$ University of East Anglia, Norwich, UK

10.1136/bmjspcare-2019-HUKNC.174

Introduction The Support Needs Approach for Patients (SNAP) is a complex intervention to facilitate delivery of person-centred care in progressive conditions, such as chronic obstructive pulmonary disease (COPD). SNAP consists of:

1. The SNAP tool: designed for purpose (Gardener, Ewing, \& Farquhar, 2019; Gardener, Ewing, Kuhn \& Farquhar, 2018;
Gardener, Ewing \& Farquhar, 2018) comprising 15 evidencebased validated questions to help patients consider areas where they may need more support;

2. The SNAP approach: a needs-led conversation between the patient and healthcare professional (HCP) to express, prioritise and address unmet support needs identified through the patient-completed tool.

Feasibility and acceptability of the SNAP intervention now needs exploring in clinical practice.

Methods HCPs from three care settings (primary, community and secondary) were recruited to the SNAP pilot in the East of England. SNAP-trained HCPs implemented SNAP with patients with COPD $(n=56)$ over a four month period and participated in monthly qualitative interviews to monitor tool use and identify modifiable barriers to SNAP delivery. Purposively sampled patients $(n=20)$ who received the intervention were interviewed about their experience. End of pilot focus groups with participating HCPs explored barriers and facilitators to delivering SNAP in clinical practice. Normalisation Process Theory (May \& Finch, 2009) guided data collection and analysis.

Results SNAP was considered acceptable and feasible by HCPs and acceptable to patients with COPD, with some variation by setting. This variation may relate to the nature of the setting and/or HCP investment in SNAP which may compromise intervention fidelity. HCPs identified three challenges for implementation: 1) limited consultation time, 2) delivering SNAP to non-English speakers, and 3) belief amongst some HCPs that they 'already do this.' SNAP enabled new needs-led conversations (including future care conversations) and consequent actions, even where patient-HCP relationships were well-established.

Conclusion SNAP appears acceptable and feasible for use in practice with potential benefits to patients with COPD across a range of care settings. Findings are informing SNAP training refinement and indicate that formal evaluation of SNAP is warranted.

\section{P-153 A HOSPICE-WIDE PROJECT TO DEVELOP AND SUPPORT MULTI-PROFESSIONAL PARTICIPATION IN CLINICAL AUDIT}

Jean Levy, Rosie Chester, Chris Doyle, Gordon Glen, Joy Ross. St Christopher's Hospice, London, UK

10.1136/bmjspcare-2019-HUKNC.175

Background Clinical audit within the organisation was perceived as piecemeal and lacking focus: staff lacked motivation and/or confidence to carry out audits. A new programme was devised to inspire and enthuse staff to examine areas of interest, encourage ownership of improvement activities, build staff skills in audit, and to boost multi-professional team working. A project was established, involving clinical, managerial and other staff, focusing on a particular area (discharge).

Aim The project aimed to achieve:

- Greater staff involvement in clinical audit;

- A training programme to improve staff knowledge of and interest in clinical audit, and address the organisation's audit priorities;

- Identification of other priorities for quality improvement projects; 
- Support for completion of specific audits.

Method A project lead oversaw and drove the project by:

- Developing and delivering training courses and materials;

- Establishing quality improvement projects;

- Supporting staff to complete those projects over a three month period.

Staff were invited to participate by their managers. A series of six workshops covered the stages of audit. The overarching topic was discussed and broken down into different areas for examination by groups. The groups were supported with their individual projects.

Results Six projects included communication, information sharing and timings. Participants agreed items of most concern/ interest, learned where to find evidence and standards; how to design tools, collect, analyse and present data. Participants enthusiastically presented results and identified areas for improvement together. Groups were multidisciplinary and demonstrated benefit from examining processes objectively.

Discussion/conclusions Support through tailored workshops and individual advice meant each group completed their project and participants were enthusiastic in championing changes to improve care. Confidence increased and multi-professional groups worked well together. Further refinement could improve the programme which is to be repeated with a different focus. Lessons learnt included ensuring good communication about the programme and getting the timings of workshops right.

\section{P-154 IMPROVING QUALITY IN A HOSPICE}

Anne Bisset-Smith, Jane Naismith. St. Joseph's Hospice, London, UK

\subsection{6/bmjspcare-2019-HUKNC.176}

Background Previously, the hospice instituted changes in the light of national guidance and themes from incidents, but the manner in which these changes were implemented lacked quantitative proof that the change was an improvement. Also, insufficient attention was paid to defining the root cause of an issue and the possibility of unintended negative consequences.

Aim For any clinical improvements to be pursued using the Institute for Healthcare Improvement (IHI) model of quality improvement (QI). This includes clearly defining the aim, using a baseline measure to clarify the issue, putting a specific intervention into place and using outcome and balancing measures to check whether the change constituted an improvement.

Method A 'Quality Improvement and Clinical Governance Lead' post was created and filled in November 2018.

The post holder:

- Delivers QI teaching on a monthly basis, with the aim of $60 \%$ of clinical staff being trained by March 2020;

- Attends all clinical incident review meetings and assists the team to address themes using QI methodology;

- Oversees all patient feedback in the same manner;

- Supports individuals and teams to run QI projects.

Results By June 2019, 17 members of staff had been trained. The initial session was delivered to multi-professional managers and then rolled out to all clinical staff. The feedback from the sessions was positive;

'Step by step process to support QI'.

'Will enable me to measure QI improvement'.

'Helped to organise some of my own ideas and study my own PDSA cycle'.

This training, together with the presence of the QI lead in all patient quality meetings, has led to proposed changes across the hospice being designed and evaluated using the QI model.

Conclusions The use of QI methodology has led to more sustainable and effective interventions and has given the hospice clearer evidence of our improvement journey.

\section{P-155 EFFECTS OF A WEEKLY HOSPICE BASED EXERCISE GROUP ON TIMED UP AND GO (TUG) AND PATIENT SATISFACTION}

Miriam Lemon. Wirral Hospice St John's, Higher Bebington, UK

\subsection{6/bmjspcare-2019-HUKNC.177}

Background Patients with life-limiting illnesses often experience functional impairment, resulting in poorer quality of life (QOL). Exercise can improve physical, emotional and psychological wellbeing which help maintain independence and improve QOL (Wittry, Lan \& McNalley, 2018). A successful chair-based exercise group is well established, however, a need was identified for a more challenging group for patients with a higher level of functional mobility.

Aims of the project To establish an exercise group to provide a relaxed and sociable environment to promote strength and balance exercises. To investigate changes in mobility after attending a weekly exercise group. To investigate patient satisfaction for the service. To empower patients to self-manage their condition and continue with exercise at home.

Methods Patients were assessed using the TUG outcome measure (Podsiadlo \& Richardson, 1991) on initial assessment. Patients attended a weekly exercise group under the supervision of a physiotherapist with volunteer support, involving exercises to improve strength and balance. TUG was repeated at intervals of four attendances and a satisfaction questionnaire was completed at the end of eight sessions.

Results All attendees showed improvements in TUG with mean reduction in walking time of 3.64 seconds (range: 0.25-8.46 seconds). After four exercise sessions 71\% of patients had a TUG score of 14 seconds or less - suggesting a lower risk of falls. Satisfaction with the group was high with many positive themes being reiterated including selfesteem, self-confidence and motivation; and no negative feedback received. $100 \%$ of responders enjoyed the exercise group and found it relevant to them. 100\% agreed or strongly agreed that the exercise was at an appropriate level and alternative exercises were suggested when necessary for individual ability.

Conclusion The exercise group has proved to be a positive addition to hospice services and demonstrated that simple strength and balance exercises are effective in improving both physical and psychological health in this patient group. 